\title{
Brittle fracture model parameter estimation for irradiated BCC material through dislocation based crystal plasticity model
}

\author{
Kulbir Singh \\ Indira Gandhi Centre for Atomic Research, HBNI, Kalpakkam, India - 603102 \\ kulbir@igcar.gov.in \\ C. Robertson \\ DEN-Service de Recherches Metallurgiques Appliquees, CEA, Universite Paris-Saclay, Gif-sur-Yvette, France \\ christian.robertson@cea.fr
}

A.K. Bhaduri

Indira Gandhi Centre for Atomic Research, HBNI, Kalpakkam, India - 603102.

bhaduri@igcar.gov.in

\begin{abstract}
Radiation effects lead to a significant reduction in ductility during the life of the components used in nuclear reactors. A sharp change in fracture toughness at a lower temperature in Body Centered Cubic (BCC) materials as compared to Face Centered Cubic (FCC) materials is a major concern restricting their application in nuclear reactors in spite of having better thermal properties, excellent resistance to helium embrittlement and void swelling under higher dpa levels. In the present paper, such a strong temperature dependence of strain rate and flow stress in BCC materials is investigated numerically for both non-irradiated and irradiated conditions. The BCC materials subjected to radiation would undergo embrittlement, which raises the ductile to brittle transition (DBT) temperature up to or above the room temperature. In view of dislocations mobility being a fundamental property to determine the plastic behavior, the dislocations based material model is proposed, which has the physical rather than phenomenological basis. This material model accounts for both thermally activated and athermal regime dislocation mobilities in BCC materials and is capable of predicting the effect of irradiation-induced defects on the mobility of the dislocations which in turn directly affect the behavior of such materials. The relative change in the stress field due to the presence of irradiation defects in comparison to the nonirradiated case provides valuable input for brittle fracture model to develop advanced materials for nuclear application.
\end{abstract}

KEYWORDS. Irradiation defects; Crystal plasticity; Dislocation mobility.

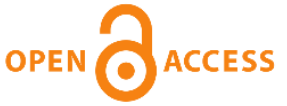

Citation: Singh, Kulbir, Robertson, C, Bhaduri, A.K, Brittle fracture model parameter estimation for irradiated BCC material through dislocation based crystal plasticity model, Frattura ed Integrità Strutturale, 50 (2019) 319-330.

Received: 30.11 .2018

Accepted: 12.05 .2019

Published: 01.07.2019

Copyright: (C) 2019 This is an open access article under the terms of the CC-BY 4.0, which permits unrestricted use, distribution, and reproduction in any medium, provided the original author and source are credited. 


\section{INTRODUCTION}

$\mathrm{D}$ efect clusters (interstitial \& vacancies) produced during irradiation strongly affects the mechanical properties of the structural materials. In irradiated BCC materials, it has been observed that dislocation-loops (interstitial type) are primary defects produced [1]. The interaction of these defects with dislocations controls the material hardening and embrittlement behavior. For the given material, chemical composition and irradiation flux, the number of dislocationloops $\left(N_{i r r}\right)$ formed, and their diameter $\left(d_{i r}\right)$ is majorly dependent upon the irradiation dose and temperature respectively $[2,3]$. Furthermore, in BCC materials, plasticity is strongly temperature dependent and could be defined according to low and high temperature regimes named as a thermal and athermal regime, respectively $[4,5]$. In thermal regime, the plasticity in BCC materials is temperature dependent (kink pair formation) whereas in athermal regime it is mostly independent of temperature. To study such behavior, crystal plasticity models within the finite element methods have become an important tool for material development and continuum mechanics based evaluation of materials [6].

Development of suitable model based on the available experimental data could facilitate in simulating irradiation effects, which otherwise involve considerable time and cost to conduct an experiment in a controlled environment. Dislocation based crystal plasticity can be used at the continuum scale to model the evolution of various crystallographic variables on each slip system to account for heterogeneous plastic strain development in crystal. This will allow to include the physical definition of different plasticity mechanisms depending upon the wide range of temperature and strain rates along with dislocations and dislocation-loops interaction. Crystal plasticity models are developed with dislocation density as internal state variable to investigate the plasticity in BCC materials by Zerilli et al. [7], Armstrong et al. [8], Kubin et al. [9], Stainier et al. [10], Ma et al. [11], Alankar et al. [12], Monnet at al. [13] and Cereceda et al. [14]. Also, attempts have been made to account for irradiation effects on the plasticity of BCC materials. Dispersed barrier hardening model has been used to describe the irradiation dose and temperature effect on the yield stress of the materials [15]. Dislocation multiplication due to their pinning with irradiation-induced defects [16] and assuming the irradiation defects as shearable obstacles for FCC materials [17] is used to study the irradiation defects. In the current work, dislocation density based crystal plasticity material model for BCC material is used to account for temperature dependent plasticity in non-irradiated and irradiated materials. The radiation induced defects are considered by incorporating strength based defect interaction with dislocations and also their impact on the mobile dislocation density in addition to total dislocation density.

The developed dislocation based material model takes into account the irradiation defects along with temperature dependent plasticity behavior of BCC materials [7-9]. Constitutive equations adapted (discussed in subsequent sections) to define thermal and as well as athermal regime assist in determining the plasticity behavior for a wide range of temperature and strain rates. The radiation induced defects are accounted by incorporating strength based defect interaction with dislocations and also their impact on the mobile dislocation density in addition to total dislocation density. The model can be used to analyze the effect of irradiation-induced dislocation-loop size and number density on the BCC material, which indirectly simulates the various irradiation conditions on single-crystal.

In the present work, the different irradiation conditions are investigated, and results are post-processed to estimate the relative change in the sub-crystalline stress distribution in the form of the Weibull parameters. These parameters will serve as valuable input for the Microstructure Informed Brittle Fracture (MIBF) model to study the effect of various irradiation condition on the fracture response of the BCC materials. The MIBF model is based on a local approach and accounts for microstructural aspects of material along with behavioral law and fracture mechanics [10]. It relies on crystal plasticity results in terms of heterogeneity of the stress field at the microstructure scale to study the compact tension (CT) specimen behavior under applied loading. An elementary volume (zone near the crack tip) consists of cleavage triggering sites like carbides having specific size distribution depending upon materials composition. Each carbide is a potential site for the cleavage and taken as the point of nucleation. The rupture (propagation) will be triggered at such sites according to the Griffith criterion (local stress and critical stress). Finally, the MIBF model provides the failure/rupture probability based on the carbide size distribution and local stress distribution obtained from crystal plasticity. These results are transformed to estimate the variation in toughness as the function of temperature.

The dislocation based material model, simulation results, and the effect of various irradiation conditions on the Weibull parameters to be subsequently used in the MIBF model are highlighted and discussed in the following sections. 


\section{MATERIAL MODEL}

he dislocation based material model is developed for BCC materials accounting for 12 slips systems ( $\{110\}$ $1 / 2<111>$ ). This material model accounts for temperature dependent plasticity along with the influence of irradiation-induced defects in the form of interstitials loops. The following subsections discuss the various constitutive equations used in the material model.

\section{Temperature dependent plasticity}

The temperature dependence of plasticity in BCC materials is accounted by two independent formulations meant for thermal and athermal regimes. Dislocation velocity in thermal regime is governed by kink pair nucleation [7-9] and is taken as a function of effective shear stress and temperature value. Subsequently, using Orowan's relation $\dot{\gamma}=\rho_{m} b v$ to relate mobile dislocation density $\left(\rho_{m}\right)$ and dislocation velocity $(v)$ with strain rate $(\dot{\gamma})$, the thermal regime strain rate is expressed as

$$
\dot{\gamma}_{\text {Thermal }}=\rho_{m} b \frac{8 \pi \tau_{e f f}^{2}}{\mu B} \exp \left[-\frac{\Delta H_{0}}{k_{B} T}\left(1-\left[\frac{\tau_{e f f}}{\tau_{0}}\right]^{p}\right)^{q}\right] l_{s}
$$

where p \& q are material constants. Eqn. 1 gives the dependence of strain rate on temperature, effective resolved shear stress and, an average length of screw dislocation segment. Athermal regime's dislocation mobility is controlled by the velocity of edge dislocations, which leads to effective resolved shear stress dependence. However, a minor thermal component is observed due to the presence of thermally activated jog drag. For the athermal regime, the following expression is adapted for strain rate to ensure the accurate coupling between strain rate sensitive macroscopic response and rate sensitive microscopic evolution of critical shear stress [9].

$$
\dot{\gamma}_{\text {Atbermal }}=\rho_{m} b \frac{8 \pi \tau_{e f f}^{2}}{\mu B} \exp \left[-\frac{\Delta H_{0}}{k_{B} T}\left(1-\left[\frac{\tau_{e f f}}{\tau_{0}}\right]^{p}\right)^{q}\right] X_{\infty}
$$

$X_{\infty}$ represents the distance swept by a kink pair before its annihilation with another kink pair along with an infinitely long screw dislocation. Various terms in Eqn. (2) are defined as:

$$
X_{\infty}=2 \sqrt{v_{k} / J}, \quad v_{k}=\tau_{e f f} b / B \quad \text { and } \quad J=\frac{8 \pi \tau_{e f f}^{2}}{\mu B h} \exp \left[-\frac{\Delta H_{0}}{k_{B} T}\left(1-\left[\frac{\tau_{e f f}}{\tau_{0}}\right]^{p}\right)^{q}\right]
$$

Based on time spent in each regime, the harmonic mean is calculated from individual strain rates representing each regime to obtain the generalized expression for the strain rate $[9,11]$.

$$
\frac{1}{\dot{\gamma}_{\text {Total }}}=\frac{1}{\dot{\gamma}_{\text {Thermal }}}+\frac{1}{\dot{\gamma}_{\text {Athermal }}} \quad \text { or } \quad \dot{\gamma}_{\text {Total }}=\frac{l_{s} X_{\infty}}{l_{s}+X_{\infty}}
$$

This expression represents the flow rule for the material model. At lower temperature range (thermal regime), the value of $l_{s}$ is smaller than $X_{\infty}$ which makes total strain rate dependent upon the $l_{s}$, whereas in athermal regime $X_{\infty}$ reduces as compared to $l_{s}$, making total strain rate as $X_{\infty}$ dependent.

\section{Hardening and obstacles strengthening}

Hardening in the material is considered, which arises due to resistance to dislocation motion provided by the obstacles cutting the slip planes. These obstacles in the form of forest dislocation can multiply during plastic deformation and increase 
slip resistance. The shear stress must reach a critical value called critical resolved shear stress $\left(\tau_{c}\right)$ for overcoming the opposition to dislocation motion, hence effective shear stress $\tau_{\text {eff }}$ which is the stress required to impart velocity $v$ to the screw dislocation segment and can be expressed as:

$$
\tau_{e f f}=\left|\tau_{R S S}\right|-\tau_{c}
$$

The $\tau_{c}$ is defined as $\tau_{c}{ }^{2}=\tau_{\text {self }}{ }^{2}+\tau_{L T}{ }^{2}, \tau_{\text {self }}$ is stress due to an interaction between all dislocations present on the same slip system, and $\tau_{L T}$ is line tension resistance on dislocation. Critical stress on any slip system is dependent upon the dislocation density and strength of interaction of all systems with respect to the reference system [13,14]. Based on relative strength between each slip system, the matrix form of interaction coefficients $\left[\alpha_{A F}\right]$ is adapted [15-17]. [ $\left.\propto_{A F}\right]$ is an interaction matrix with size dependent upon the number of interacting slip systems considered, and each coefficient represents the strength of the interaction. In current work, 12 slip systems are considered, which can be defined with interaction matrix (size $\left[\propto_{A F}\right]=12 \times 12$ ) having six independent coefficients. The value of $\tau_{\text {self }}$ is determined based on the interaction strength between the same slip system $\propto_{\text {self }}$ and is expressed as $\tau_{\text {self }}=\mu b \sqrt{\alpha_{\text {self }} \rho_{\text {self }}}$.

The average length of screw dislocation (in Eqn. 1) is directly dependent upon the distance between forest obstacles encountered by it. These forest obstacles can be in the form of dislocations, particles, or irradiation defects. The average length of a screw dislocation is estimated based on the line tension model using obstacle spacing, obstacle strength, and corresponding obstacle number density [7-9, 11]. Following are the equations used to estimate the average length of screw dislocation when it encounters an obstacle:

Obstacle spacing

The average length of screw dislocation

Average obstacle strength

A minimum value of the average length of screw dislocation

Radius of curvature

$$
\lambda=\frac{1}{\min \left[\sqrt{\rho_{o b s}^{A} ;\left(2 R_{s}+D\right) \rho_{o b s}^{A}}\right]}-D
$$

$$
\alpha=\sqrt{\sum \alpha^{A F} \frac{\rho^{F}}{\rho_{o b s}^{A}}}
$$

$$
l_{\text {min }}=f_{c} L_{c}
$$

( $\mathrm{fc}$ is constant, estimated to be six based on fitting with experimental results for activation volume)

$$
R_{s}=\mu b / 2 \tau_{\text {eff }}
$$

The $\tau_{L T}$ value becomes significant and contributes to Eqn. 4 for smaller obstacle spacing [11]. This is defined based on the radius of curvature as:

$$
\tau_{L T}=\frac{\mu b}{2 R_{c}}-\frac{\mu b}{2 R_{s}}
$$

where $R_{c}=\lambda / 2 \alpha$ and $\alpha$ depend upon the forest and obstacle dislocation densities. Effect of irradiation defects is taken appropriately based on their strength and number density in calculation of the above internal variables in the constitutive model [9].

Irradiation defects are treated as obstacles to dislocation motion and hence induce irradiation hardening. Because of the generation of distinct internal stress due to complex structure generation in dislocation-irradiation defect interaction, a 
correction factor $f_{l}$ is introduced [9]. This factor scales with average spacing due to the presence of irradiation defects and is multiplied with total strain rate value given in Eqn. 3 (accounting an effect on velocity).

$$
f_{l}=\min \left(\frac{l_{s}}{l_{\min }}\left[\sqrt{\frac{\rho_{m}}{2 N_{i r r} d_{i r r}}}\right], 1\right)
$$

\section{Dislocations and dislocation-loops density evolution}

Strain dependence is used to define the dislocation density evolution based on storage (leads to an increase in flow stress) and annihilation (leads to dynamic recovery) phenomenon of dislocation as [11, 18]:

$$
\frac{d \rho}{d \gamma}=\frac{1}{b}\left[\frac{1}{\Lambda}-y \rho\right]
$$

$\Lambda$ is dislocation mean free path defined as $\frac{1}{\Lambda}=\left(1-\frac{\tau_{\text {eff }}}{\tau_{0}}\right) \frac{\sqrt{\alpha_{\text {self }} \rho^{A}}}{K_{\text {self }}}+\frac{\alpha^{A} \lambda^{A} \rho_{\text {obs }}{ }^{A}}{K_{\text {forest }}} \quad$ and $y$ is temperature dependent material parameter accounting for dynamic recovery expressed as $\frac{1}{y}=\frac{1}{y_{p r o p}}+\frac{2 \pi \tau_{e f f}}{\mu b}$.

Temperature dependence of $y$ is accounted by using a harmonic average of critical distance in thermal $\left(\frac{\mu b}{2 \pi \tau_{e f f}}\right)$ and athermal regime $\left(y_{\text {prop }}\right)$.

For irradiated materials, the current investigation focuses on dislocation loops (sessile), which are primary irradiationinduced defects. It is assumed that the material has pre-known dislocation loops density $\left(\rho_{i r r}=N_{i r r} d_{i r r}\right)$ due to radiation exposure. Further, these defects will interact with the dissociations to induce strain hardening. A gradual reduction in the irradiation defect density on the active slip plane due to their interaction with mobile dislocations is accounted. Rate of change of $\rho_{i r r}[8]$ is defined as:

$$
\dot{\rho}_{i r r}=-\xi \rho_{i r r} \dot{\gamma}
$$

with $\xi$ as a loop annihilation parameter. Increase in the value of $\rho_{t o t}$ (accounted in Eqn. 12) and $\rho_{m}$ is generally observed due to the presence of irradiation defects in the material $[12,19]$. The increase in $\rho_{m}$ may be attributed to the interaction of irradiation defects with screw dislocation lines. The rate of increase of mobile dislocations due to interaction with irradiation defects is expressed as:

$$
\dot{\rho}_{m}=\frac{\kappa \xi}{r_{0}} \rho_{i r r(t)} \dot{\gamma}
$$

where $\kappa$ is the number of screw-loop interaction which produces dislocation source before irradiation loop's removal from lath and $r_{0}$ is an initial ratio of $\rho_{i r r}$ over $\rho_{m}$. The resistance against the dislocation motion is dependent upon the size (increases for higher defect size) of the defects (loops) along with their number density. Size effect of irradiation defects is incorporated by introducing size factor $f_{\text {size }}$ [9] defined as:

$$
f_{\text {size }}=\max \left(s_{r} \times \frac{d_{i r r}}{d_{r}}, 1\right) \quad \text { with } \quad s_{r}=\min \left(\frac{N_{i r r} d_{i r r}}{\left(N_{i r r} d_{i r r}\right)_{\text {lim }}}, 1\right)
$$


$d_{r}$ is the reference diameter (taken as $\left.2 \mathrm{~nm}\right),\left(N_{i r} d_{i r}\right)_{\text {lim }}$ is the material parameter defining its saturated value for increasing dose. The factor $f_{\text {size }}$ is multiplied with Eqn. 14, this incorporates the defect size dependence of the dislocation velocity. The various material parameters $[7-9,11]$ used in the material model are listed in Tab. I.

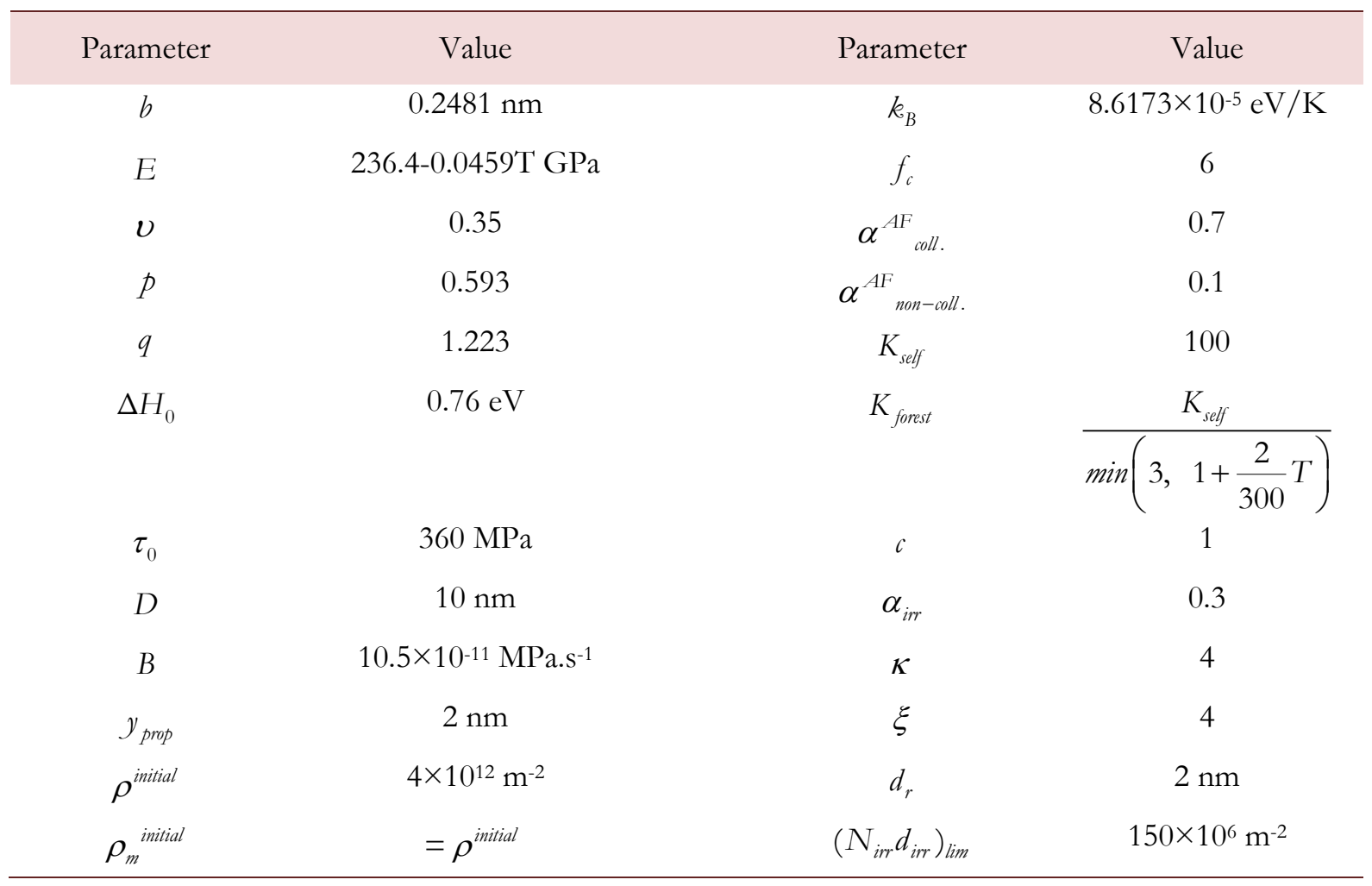

Table I: Various material parameters considered in material model.

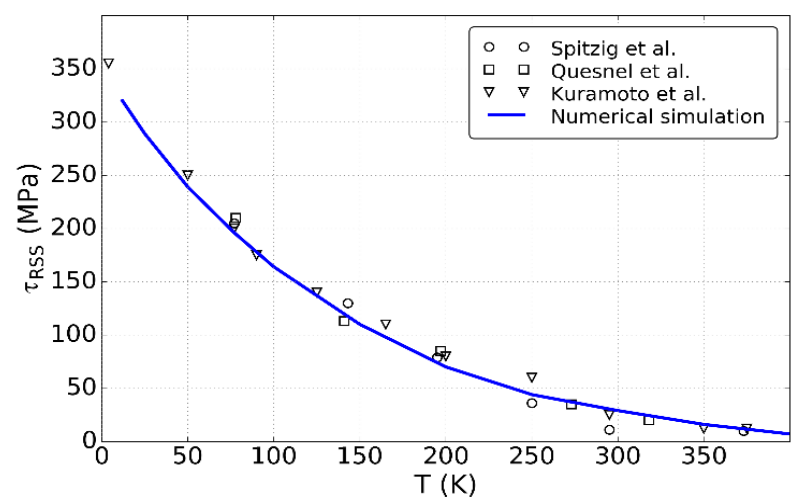

Figure 1: Validation of a material model with experimental results: resolved shear stress variation as a function of temperature.

\section{Finite element formulation}

The constitutive equations discussed in previous sections encompass the behavior of BCC materials under both nonirradiated and irradiated conditions. Flow rule is defined by Eqn. 1, 2 \& 3, Eqn. 4 to 10 define the hardening behavior, Eqn. 12 defines the evolution of dislocation density and Eqn. 13 and Eqn. 14 defines the evolution of irradiation defects and mobile dislocation density, respectively. These equations are solved at each increment for 12 slip systems to estimate the plasticity behavior. The constitutive equations are formulated with finite strain formulation using the MFRONT interface [20] and subsequently solved with finite element solver. The results are validated with available experimental data in terms of resolved shear stress [21-23]. The material model predicts satisfactorily the temperature dependence of plasticity in BCC materials (shown in Fig. 1). For irradiated material, it is also capable of predicting the effect of irradiation defects for different size and number densities, highlighting the influence on hardening and strain localization [9]. 


\section{Application of the material model}

In the present work, a relative change in local stress at the microstructure level is predicted, and the same will be used as input to the MIBF model. Predicted stress field would assist in estimating the rupture probability based on a local approach. MIBF model relies on crystal plasticity results in terms of heterogeneity of the stress field at the microstructure scale [10]. The concept, theory, and application of MIBF model are discussed in detail elsewhere [10, 24-27]. Generally, a compact tension (CT) specimen is modeled in the FEA framework to apply the MIBF. An elementary volume (zone near the crack tip) consists of cleavage triggering sites like carbides having specific size distribution depending upon materials composition. Each carbide is a potential site for the cleavage and taken as the point of nucleation. The rupture (propagation) will be triggered at such sites according to the Griffith criterion (local stress and critical stress). Finally, the MIBF model provides the failure/rupture probability based on the carbide size distribution and local stress distribution obtained from crystal plasticity. MIBF model is capable of reproducing the variation in toughness with temperature and irradiation without arbitrarily introducing a change in cleavage critical stress.

To assess the effect of various irradiation conditions at the microstructure level, a unit cell model is analyzed at $300 \mathrm{~K}$ for non-irradiated and irradiated material cases. In the unit cell, the small inclusion of radius $2 \mu \mathrm{m}$ (with and without crack) present at the center of the unit cell is envisaged (unit cell with inclusion case, shown in the Fig. 2). This is to account for fracture behavior dependency upon the defects present at the microscale. Mostly, inclusions (sulphides, oxides, nitrides, etc.) which act as a crack initiator are harder than the surrounding material. In the present study, the inclusion is assumed to be elastic and have E value $300 \mathrm{GPa}$. The direction of loading is oriented such that it inclines with [-149] direction of the crystallographic axis. The displacement controlled loading in the form of $10 \%$ global strain is applied at one face of the unit cell, while the opposite face is restricted.
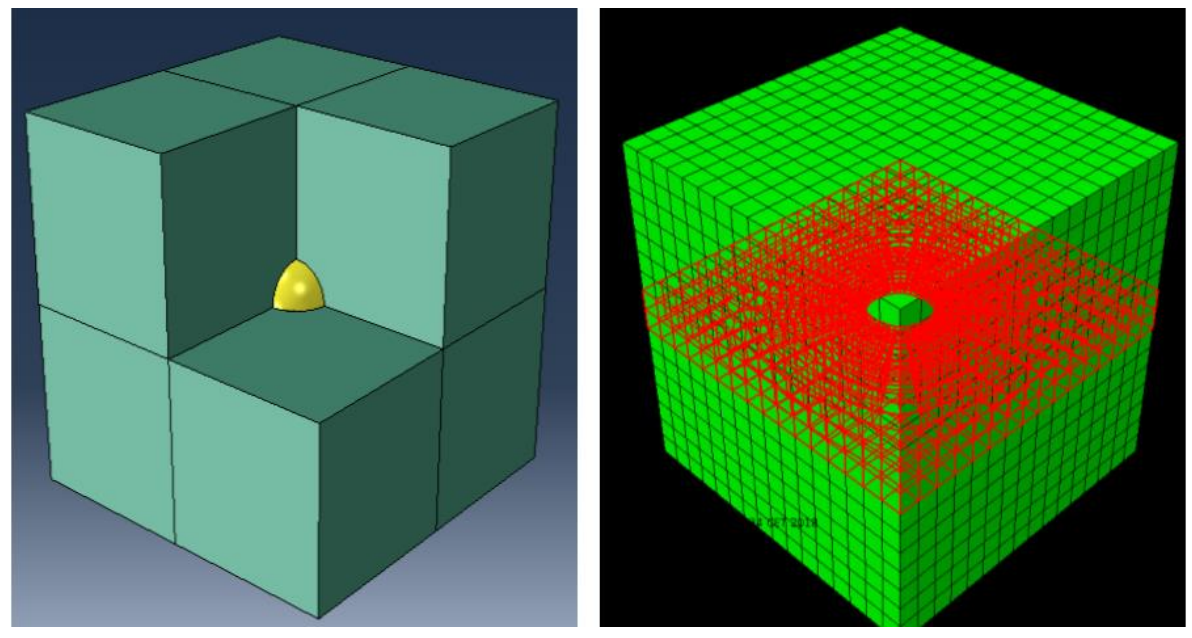

Figure 2: Unit cell model with inclusion, the meshed model with a highlighted area of consideration for Weibull distribution's parameter estimation

\section{RESULTS AND DISCUSSION}

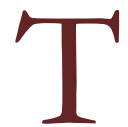

he various irradiation conditions are analyzed corresponding to different defect size and number density. Each set of defect size $(2 \mathrm{~nm}, 5 \mathrm{~nm}, 10 \mathrm{~nm}, 15 \mathrm{~nm}, 25 \mathrm{~nm}, 50 \mathrm{~nm})$ and their number density $\left(1 \times 10^{12} \mathrm{~mm}^{-3}, 2 \times 10^{12} \mathrm{~mm}^{-3}\right.$, $4 \times 10^{12} \mathrm{~mm}^{-3} 6 \times 10^{12} \mathrm{~mm}^{-3}, \quad 8 \times 10^{12} \mathrm{~mm}^{-3}, \quad 10 \times 10^{12} \mathrm{~mm}^{-3}, 12 \times 10^{12} \mathrm{~mm}^{-3}, 14 \times 10^{12} \mathrm{~mm}^{-3}$ ) represents the particular irradiation condition in terms of irradiation temperature and dose. The simulation results obtained are further post-processed to get the frequency distribution of the equivalent stress value in the unit cell. This is used in the MIBF model in the form of Weibull distribution to calculate the failure probability of toughness specimen.

Further, an effect of irradiation on local stress distribution is estimated in terms of relative change in parameters of Weibull distribution with respect to the non-irradiated case. This relative change in parameters as a function of irradiation defect size and number density assist in estimating the fracture toughness of irradiated materials. The rate of change of parameters of Weibull distribution due to irradiation corresponds to the rate of change of the local stress distribution in the elementary volume due to the respective irradiation condition. The purpose of the current work is to solely provide an effect of 
irradiation conditions relative to non-irradiated conditions. The elemental area normalization is carried out for calculation of frequency distribution of the equivalent stress in the zone highlighted in Fig. 2. The frequency data is extracted for each case, and Weibull distribution is fitted which is represented by:

$$
f(\sigma)=\frac{\beta}{\eta}\left(\frac{\sigma-\gamma_{w}}{\eta}\right)^{\beta-1} \exp { }^{-\left(\frac{\sigma-\gamma_{w}}{\eta}\right)^{\beta}}
$$

where $\beta$ is the shape parameter, $\eta$ is the scale parameter, and $\gamma_{w}$ is the position parameter (zero in this case).

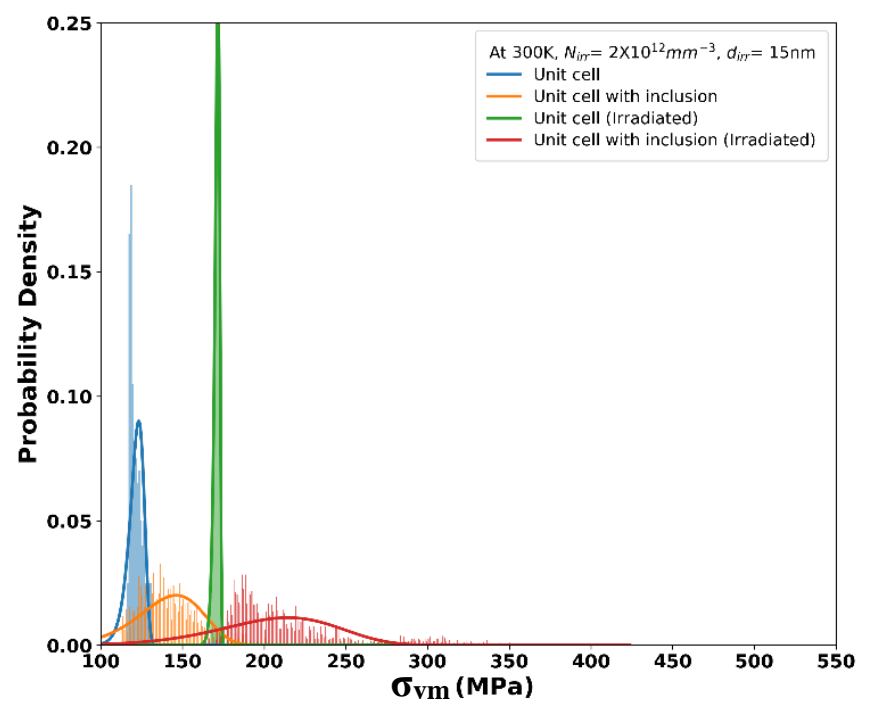

(a)

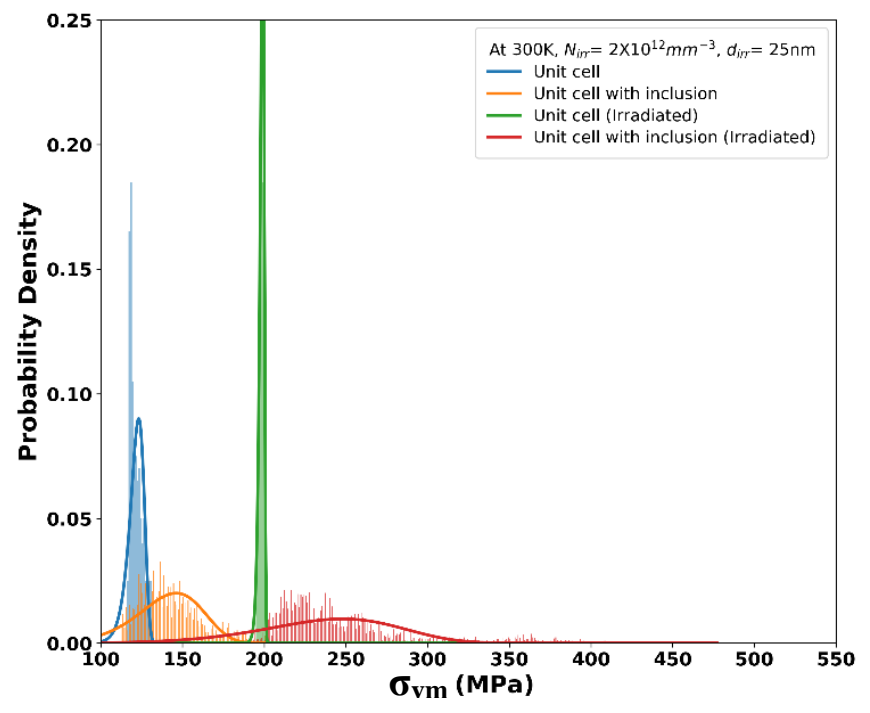

(c)

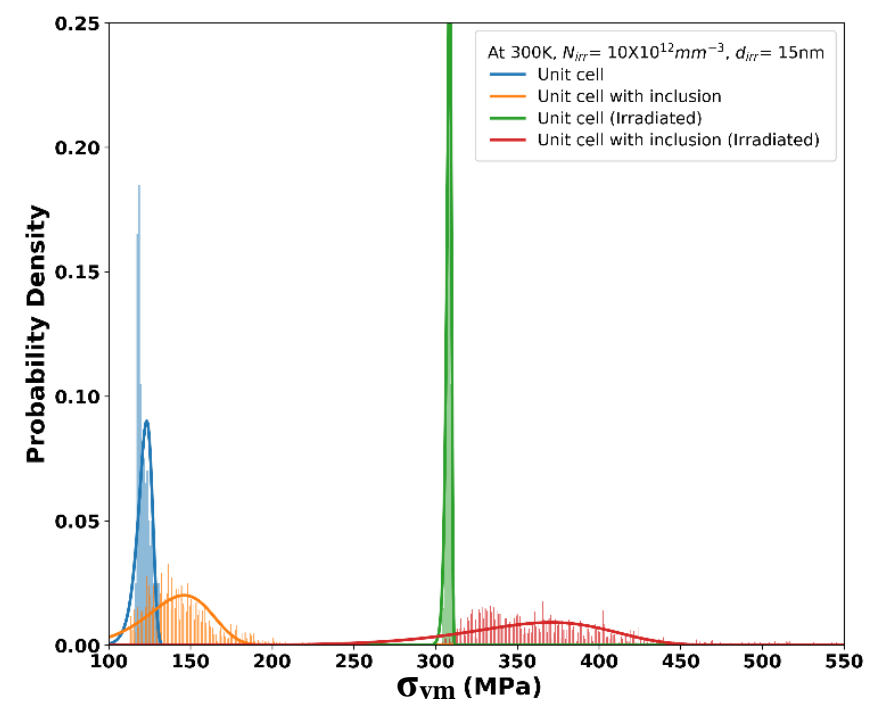

(b)

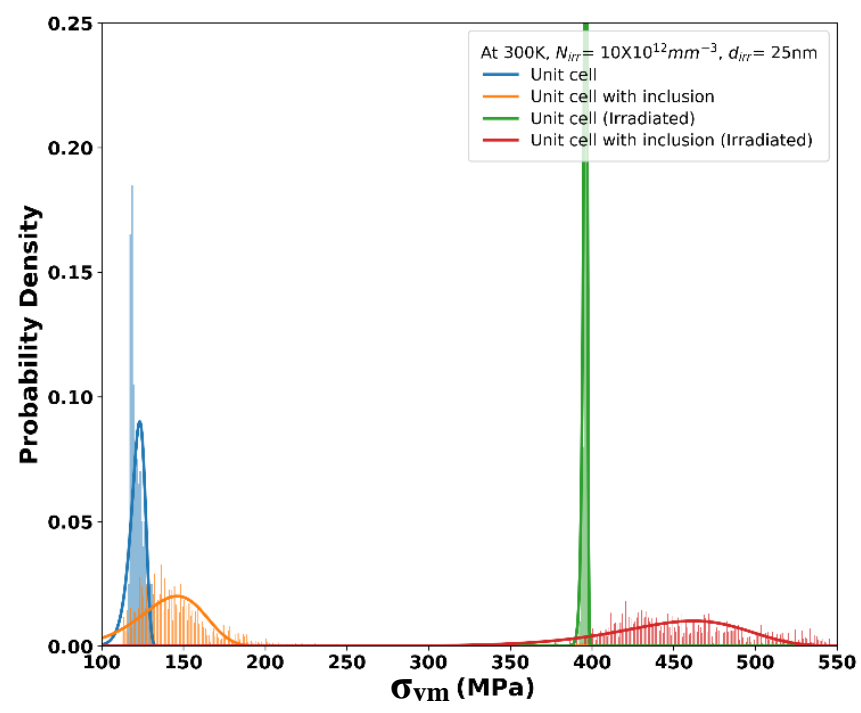

(d)

Figure 3: Probability density of equivalent stress for both irradiated and non-irradiated conditions at $300 \mathrm{~K}$, with corresponding fitted Weibull distribution: Results of irradiation condition with a) $N_{i r r}=2.0 \times 10^{12} \mathrm{~mm}^{-3}$ and $d_{i r r}=15 \mathrm{~nm}$, b) $N_{i r r}=10.0 \times 10^{12}$ $\mathrm{mm}^{-3}$ and $\left.d_{i r r}=15 \mathrm{~nm}, \mathrm{c}\right) N_{i r r}=2.0 \times 10^{12} \mathrm{~mm}^{-3}$ and $d_{i r r}=25 \mathrm{~nm}$ and d) $N_{i r r}=10.0 \times 10^{12} \mathrm{~mm}^{-3}$ and $d_{i r r}=25 \mathrm{~nm}$. 
The stress field (for equivalent stress) in the unit cell is transformed into probability density for the non-irradiated and irradiated case. The probability density of the local stress field in the unit cell and the corresponding Weibull distribution of the stress field in the unit cell are plotted against the equivalent stress for various irradiation conditions in Fig. 3. It shows the results for different irradiation conditions (irradiation flux and temperature). The irradiation temperature defines the size of the dislocation loop $\left(d_{i r r}\right)$, whereas the flux controls the irradiation defect number density $\left(N_{i r r}\right)$ of irradiation defects. Similar defect size (say $15 \mathrm{~nm}$ in Fig. 3a and 3b) with different irradiation defect number density $\left(N_{\text {irr }}\right)$ represents the material exposed to different flux levels but at the same temperature during irradiation. In the case of Fig. 3a and 3c, the irradiation defect density is same but differ in defect size; this depicts the material irradiated at different temperature values but same flux. Presence of irradiation defects shifts the mean stress value towards higher magnitude relative to the nonirradiated case representing the irradiation hardening. It is observed that there is a significant shift in equivalent stress towards higher magnitude for all cases of irradiated material. The direct dose dependence of the local stress field (represented by the change in Weibull distribution) due to irradiation defects is evident from the reported results. Based on the FEA results, it is found that the influence of inclusion (with or without crack) is negligible in estimating the relative change in the stress field due to the presence of irradiation defects. Moreover, the stress raising effect of the local defects like inclusion is explicitly handled by MIBF model by having the probabilistic distribution of the carbides with a crack like defects.

The Weibull parameters ( $\beta$ shape and $\eta$ scale) estimated for each irradiation conditions are presented in the form of the relative change in their value with respect to the non-irradiation case. This relative change in Weibull parameter is plotted as a function of irradiation defect number density for each defect size (shown in Fig. 4). Through this curve, it is possible to estimate the relative change in the stress field at the microstructure level for the material exposed to irradiation conditions at a specific temperature and flux level. Knowing the value of the Weibull parameters for the non-irradiated case, the parameters for irradiated material can be estimated. Through these parameters, it is viable to determine the change in local stress distribution at microstructure level for desired irradiation conditions (flux and irradiation temperature) and finally lead to predicting the fracture response under irradiated condition using MIBF model.
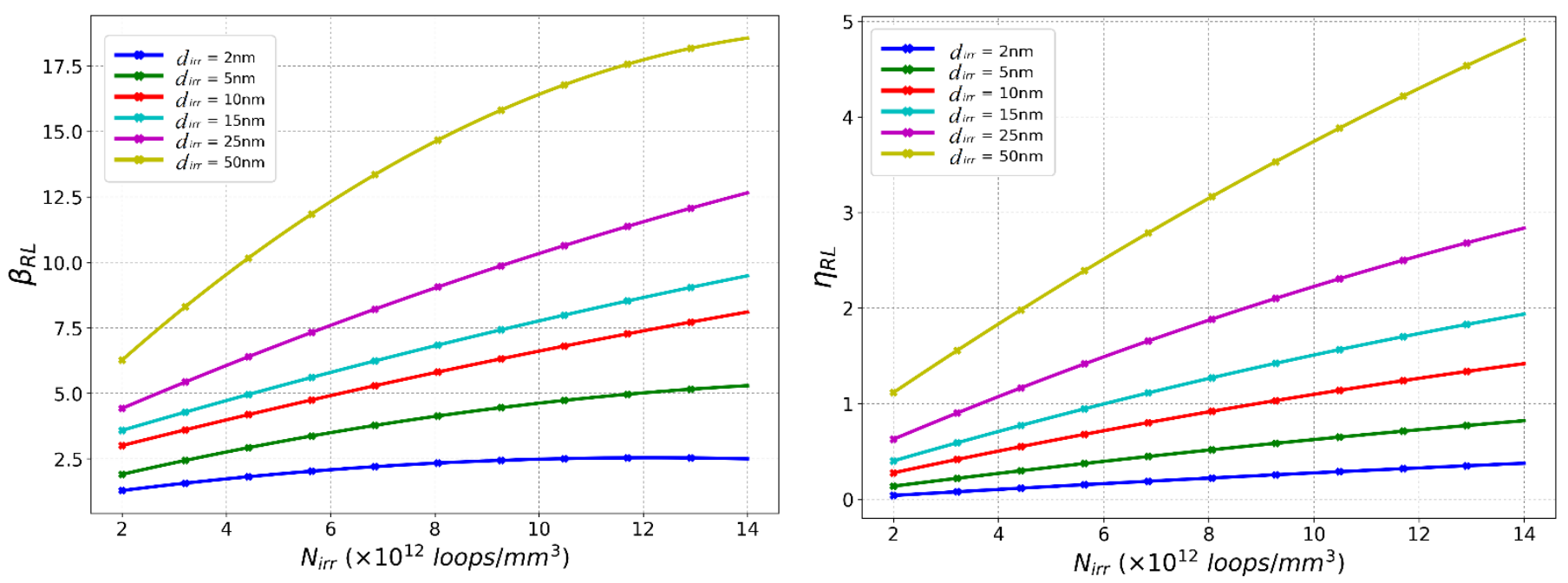

Figure 4: Relative change in Weibull distribution parameters for different irradiation defect size and number density. Subscript 'RL' represents the relative value of the corresponding parameter. For example, the relative change in the shape parameter is defined as $\left(\beta_{\text {irr }}-\beta_{\text {non-irr }}\right) / \beta_{\text {non-irr. }}$

\section{CONCLUSIONS}

$\checkmark$ he material model developed for BCC materials to define the temperature dependent plasticity along with irradiation modeling is discussed. The two different constitutive equations representing thermal and athermal regime can describe the plasticity behavior satisfactorily, which is confirmed with experimental validation. The irradiation defects are treated as barriers to the motion of the dislocation, and the evolution of various irradiation related parameters is incorporated. Presence of irradiation defects in the form of interstitial loops affects the local stress field for different irradiation conditions is presented for the unit cell model (with and without inclusion). Shifting of equivalent stress towards higher magnitude for irradiated material signifies the irradiation hardening in the material. Different irradiation conditions 
are simulated, and results are post-processed to fit the Weibull distribution, which quantifies a relative change in the local stress distribution at the microstructure level due to irradiation. Estimation of relative change in Weibull distribution parameters for irradiated material with respect to the non-irradiated case would be used in the MIBF model to predict the toughness behavior of the CT specimen of BCC material to get fracture response.

\section{ACKNOWLEDGMENT}

he present research work was jointly supported by Indira Gandhi Centre for Atomic Research-DAE, India and Jules Horowitz Reactor-CEA, France. We wish to thank Vincent Ludovic, Helfer Thomas, Pierre Forget, P.Puthiyavinayagam, K. Velusamy, S. Jalaldeen and R. Suresh Kumar for their kind help and technical support.

\section{REFERENCES}

[1] Zinkle, S.J. and Matsukawa, Y. Observation and analysis of defect cluster production and interactions with dislocations, Journal of Nuclear Materials, 329-333 (2004), pp. 88-96. DOI: 10.1016/j.jnucmat.2004.04.298.

[2] Larson B. C., Young, F. (1977). Effect of temperature on irradiation-induced dislocation loops in copper, Journal of Applied Physics, 48, pp. 880-886. DOI: 10.1063/1.323750.

[3] Hardie, Christopher D., Williams, Ceri A., Xu, S., Roberts, Steve G. (2013). Effects of irradiation temperature and dose rate on the mechanical properties of self-ion implanted $\mathrm{Fe}$ and $\mathrm{Fe}-\mathrm{Cr}$ alloys, Journal of Nuclear Materials, 439, pp. 3340. DOI: 10.1016/j.jnucmat.2013.03.052.

[4] Balasubramanian, N. (1969). The temperature dependence of the dislocation velocity-stress exponent, Scripta Metallurgica, 3, pp. 21-24. DOI: 10.1016/0036-9748(69)90171-9.

[5] Kubin, L., Devincre, B., Tang, M. (1998). Mesoscopic modelling and simulation of plasticity in fcc and bcc crystals: Dislocation intersections and mobility, Journal of Computer-Aided Materials Design, 5, pp. 31-54.

DOI: 10.1023/A:1008648120261.

[6] Roters, F., Eisenlohr, P., Hantcherli, L., Tjahjanto, D., Bieler, T., Raabe, D. (2010). Overview of constitutive laws, kinematics, homogenization and multiscale methods in crystal plasticity finite-element modeling: Theory, experiments, applications, Acta Materialia, 58, pp. 1152-1211. DOI: 10.1016/j.actamat.2009.10.058.

[7] Singh, K., Robertson, C., Bhaduri, A.K. (2017). Assessing the Irradiation Defect Induced Changes using Dislocation Based Crystal Plasticity Model for BCC Materials, Procedia Structural Integrity, 5, pp. 294-301.

DOI: $10.1016 /$ j.prostr.2017.07.136.

[8] Singh, K., Robertson, C., Bhaduri, A.K., Irradiation in BCC materials: Defect-induced changes of the effective dislocation mobility and their relation with the dose-dependent fracture response, Manuscript submitted for publication.

[9] Singh, K., Robertson, C., Bhaduri, A.K., Irradiation dose and temperature effects on BCC material with dislocation based crystal plasticity, Manuscript submitted for publication.

[10] Forget, P., Marini, B., Vincent, L. (2016). Application of local approach to fracture of an RPV steel: effect of the crystal plasticity on the critical carbide size, Procedia Structural Integrity, 2, pp. 1660-1667. DOI: 10.1016/j.prostr.2016.06.210.

[11] Monnet, G., Vincent, L., Devincre, B. (2013). Dislocation-dynamics based crystal plasticity law for the low and hightemperature deformation regimes, Acta Materialia, 61, pp. 6178-6190. DOI: 10.1016/j.actamat.2013.07.002

[12] Li, Y., Robertson, C. (2018). Irradiation defect dispersions and effective dislocation mobility in strained ferritic grains: a statistical analysis based on 3D dislocation dynamics simulations, Journal of Nuclear Materials, 504, pp. 84-93. DOI: 10.1016/j.jnucmat.2018.03.026.

[13] Taylor, G. (1934). The Mechanism of Plastic Deformation of Crystals. Part I - Theoretical', Proc. of the Royal Society of Landon. Series A, Containing Papers of Mathematical and Physical Character, 145, pp. 362-387.

DOI: 10.1098/rspa.1934.0106.

[14] Besinski, Z. (1974). Forest hardening in face centred cubic metals, Scripta Metallurgica, 8, pp. 1301-1308.

[15] Franciosi, P., Berveiller, M., Zaoui, A (1980). Latent hardening in copper and aluminium single crystals, Acta Metallurgica, 28, pp. 273-283. DOI: 10.1016/0001-6160(80)90162-5.

[16] Peirce, D., Asaro, R., Needleman, A. (1981). An analysis of nonuniform and localized deformation in ductile single crystals, Division of Engineering,Brown University, Providence. RI 02912. U.S.A.

[17] Devincre, B., Hoc, T., Kubin, L. P. (2005). Collinear interaction of dislocation and slip systems, Materials Science and Engineering A, vol 400-401, pp. 182-185. DOI: 10.1016/j.msea.2005.02.071 
[18] Estrin, Y., Mecking, H. (1984). A unified phenomenological description of work hardening and creep based on oneparameter model, Acta Metallurgica, vol 32, pp. 57-70. DOI: 10.1016/0001-6160(84)90202-5.

[19] Gururaj, K., Robertson, C., Fivel, M. (2015). Post-irradiation plastic deformation in bcc fe grains investigated by means of 3d dislocation simulations, Journal of Nuclear Materials, 459, pp. 194-204. DOI: 10.1016/j.jnucmat.2015.01.031.

[20] Thomas, H., Bruno, M., Jean-Michel, P., Maxime, S., Jerome, S., Michel, C. (2015). Introducing the open-source mfront code generator: Application to mechanical behaviours and material knowledge management within the PLEIADES fuel element modelling platform, Computers \& Mathematics with Applications, 70, pp. 994-1023.

DOI: $10.1016 /$ j.camwa.2015.06.027

[21] Spitzig, W. (1973). The effect of orientation, temperature and strain rate on deformation of Fe-0.16 wt.\% Ti single crystals, Material Science and Engineering, 12, pp. 191-202. DOI: 10.1016/0025-5416(73)90010-4.

[22] Quesnel, D., Sato, A., Meshii, M. (1975). Solution softening and hardening in the iron-carbon system, Material Science and Engineering, 18, pp. 199-208. DOI: 10.1016/0025-5416(75)90170-6.

[23] Kuramoto, E., Aono, Y., Kitajima, K. (1979). Thermally activated slip deformation of high purity iron single crystals between $4.2 \mathrm{k}$ and $300 \mathrm{k}$, Scripta Metallurgica, 13, pp. 1039-1042. DOI: 10.1016/0036-9748(79)90199-6.

[24] Vincent, L., Libert, M., Marini, B., Rey, C. (2010). Towards a modelling of RPV steel brittle fracture using crystal plasticity computations on polycrystalline aggregates, Journal of Nuclear Materials, 406, pp. 91-96.

DOI: 10.1016/j.jnucmat.2010.07.022.

[25] Vincent, L., Gelebart, L., Dakhlaoui, R., Marini, B. (2011). Stress localization in BCC polycrystals and its implications on the probability of brittle fracture, Materials Science and Engineering A, 528, pp. 5861-5870. DOI: $10.1016 /$ j.msea.2011.04.003.

[26] Libert, M., Rey, C., Vincent, L., Marini, B. (2011). Temperature dependant polycrystal model application to bainitic steel behavior under tri-axial loading in the ductile-brittle transition, International Journal of Solids and Structures, 48, pp. 2196-2208. DOI: 10.1016/j.ijsolstr.2011.03.026.

[27] N'Guyen, C.N., Barbe, F., Osipov, N., Cailletaud, G., Marini, B., Petry, C. (2012). Micromechanical local approach to brittle failure in bainite high resolution polycrystals: A short presentation, Computational Materials Science, 64, pp. 6265. DOI: 10.1016/j.commatsci.2012.03.034.

\section{NOMENCLATURE}

$T \quad$ Absolute temperature

$\rho_{m} \quad$ Mobile dislocation density $(\mathrm{m}-2)$

$\rho_{\text {obs }} \quad$ Total obstacles (forest dislocation and irradiation loops)

$\rho_{\text {self }} \quad$ Dislocation density on same slip system

$\rho^{F} \quad$ Forest dislocation density

$\rho_{\text {irr }} \quad$ Irradiation defect density

$\dot{\gamma} \quad$ Strain rate on slip system

$\alpha^{A F} \quad$ Interaction coefficient of system A with obstacle system F

$\alpha \quad$ Average obstacle strength based on a quadratic average rule

$\alpha_{\text {self }} \quad$ Obstacle strength between same slip system

$b \quad$ Magnitude of Burger's vector

$b \quad$ Distance between Peierls valleys

$B \quad$ Phonon/viscous drag coefficient

$\lambda \quad$ Average obstacle spacing $\tau_{0} \quad$ Peirels shear stress at $0 \mathrm{~K}$

$\tau_{\text {eff }} \quad$ Effective resolved shear stress on the slip plane

$\tau_{\text {RSS }} \quad$ Resolved shear stress on the slip plane

$\tau_{c} \quad$ Critical resolved shear stress

$\tau_{\text {self }} \quad$ Same slip system's dislocations induced stress

$\tau_{L T} \quad$ Line tension resistance

$\mu \quad$ Shear modulus

$\Delta H_{0} \quad$ Pair activation enthalpy at $0 \mathrm{~K}$

$k_{B} \quad$ Boltzmann constant

$N_{i r r} \quad$ Irradiation defects (interstitial loop)

number density

$d_{i r r} \quad$ Irradiation defects (interstitial loop) diameter

$X_{\infty} \quad$ Distance swept by kink pair before its annihilation

$y \quad$ Dynamic recovery parameter 


\begin{tabular}{|c|c|c|c|}
\hline $\mathrm{R}_{c}$ & $\begin{array}{l}\text { Critical radius of curvature of } \\
\text { dislocation to bypass the obstacle }\end{array}$ & $\xi$ & $\begin{array}{l}\text { Dislocation-loop annihilation } \\
\text { parameter }\end{array}$ \\
\hline $\mathrm{R}_{s}$ & $\begin{array}{l}\text { Dislocation curvature radius near } \\
\text { obstacle on slip systems }\end{array}$ & $\kappa$ & $\begin{array}{l}\text { Screw-loop interaction to produce } \\
\text { dislocations }\end{array}$ \\
\hline$l_{s}$ & $\begin{array}{l}\text { Average length of screw dislocation } \\
\text { segment on slip system s }\end{array}$ & $\beta$ & $\begin{array}{l}\text { Shape parameter of Weibull } \\
\text { distribution }\end{array}$ \\
\hline$D$ & Obstacle diameter & $\eta$ & Scale parameter of Weibull \\
\hline$L_{c}$ & Critical length of kink pair & & distribution \\
\hline \multirow[t]{2}{*}{$l_{\min }$} & Minimum/limiting value of the & $\gamma_{w}$ & $\begin{array}{l}\text { Position parameter of Weibull } \\
\text { distribution }\end{array}$ \\
\hline & $\begin{array}{l}\text { average length of screw dislocation } \\
\text { segment }\end{array}$ & $\Lambda$ & Dislocation mean free path \\
\hline & Velocity of kink & & \\
\hline
\end{tabular}

